\section{Akute Effekte von Bronchodilatatoren auf Lungenfunktion und Dyspnoe bei Patienten mit COPD}

Die chronisch-obstruktive Bronchitis und das Lungenemphysem (zusammengefasst als COPD) stellen neben dem Asthma bronchiale die häufigsten obstruktiven Atemwegserkrankungen dar [1]. Wesentliche Charakteristika der COPD sind irreversible strukturelle Änderungen der Lunge und Atemwege, die in der Konsequenz zu einer Atemwegsobstruktion führen, welche insbesondere während Exspiration zutage tritt [2].

Die Lungenfunktion, die durch den Atemstoß $\mathrm{FEV}_{1}$ charakterisiert werden kann, unterliegt in der Regel bei Patienten mit COPD nur geringen kurzfristigen Schwankungen und zeigt eine progrediente Verschlechterung. Im Vergleich dazu ist beim Asthma die kurzfristige Variabilität der Messwerte größer, jedoch die jährliche Abnahme geringer [3,4]. Bei der funktionellen Differenzierung zwischen Asthma und COPD sind die Messung der Diffusionskapazität für Kohlenmonoxid $\left(D_{\text {co }}\right)$, hochauflösende Computertomogramme [5] oder indirekte Methoden wie die Erfassung des effektiven Atemwegsdurchmessers und der Dispersion mittels inhalierter Aerosole hilfreich [6,7].

Für die exspiratorische Flussbegrenzung bei Patienten mit COPD wird ein dynamischer Kollaps der kleinen Atemwege verantwortlich gemacht, der durch den Verlust der elastischen Rückstellkräfte der Lunge bzw. Atemwege verursacht wird [8-10]. Weitere zur Atemwegsobstruktion beitragende Faktoren können eine Verengung der Atemwege durch Hypertrophie der Atemwegsmuskulatur oder Mukus sowie eine Überempfindlichkeit der Atemwege sein [11]. Die strukturellen Änderungen der Lunge und der Atemwege sind kaum einer kausalen Therapie zugänglich. Nationale und internationale Empfehlungen empfehlen daher hauptsächlich die Behandlung mit Bronchodilatatoren, in der Erwartung, durch eine stete Weitstellung der Atemwege die Folgen der dynamischen Flussbegrenzung zu verringern $[2,3,12,13]$.

Demzufolge wird auch bei Patienten mit COPD in der Regel geprüft, ob die Lungenfunktion durch Bronchodilatatoren zu verbessern ist (sog. Broncholysetest), obgleich seine Ergebnisse - wie im folgenden gezeigt - sich teilweise paradox zur klinisch-symptomatischen Wirkung der Bronchodilatatoren verhalten. Der Test erfolgt mittels Inhalation von $\beta_{2}$-Sympathomimetika oder Anticholinergika und stellt eine klinisch

Pneumologie 2000; 54: 252-255

(c) Georg Thieme Verlag Stuttgart · New York ISSN 0934-8387

\author{
C. Taube, R. A. Jörres, H. Magnussen \\ Pneumologisches Forschungsinstitut \\ am Krankenhaus Großhansdorf \\ Zentrum für Pneumologie und Thoraxchirurgie, \\ Großhansdorf
}

etablierte Methode dar, um die Wirkung derartiger Medikamente auf den Tonus der Atemwege zu erfassen; diese Wirkung steht, so ist anzunehmen, in Beziehung zum klinischen Nutzen beim individuellen Patienten. An der Bronchodilatation sind sowohl direkte als auch indirekte (z.B. über Nervenbahnen vermittelte) Wirkungen auf die glatte Muskulatur der Atemwege beteiligt [14]. Die Stärke der bronchodilatatorischen Antwort hängt von der verwendeten Substanz und Dosis ab, doch wird das Ergebnis auch von dem gewählten Messparameter bestimmt. Die Messung des bronchodilatatorischen Effektes erfolgt meist mittels Atemwegswiderstand oder Atemstoß ( $\mathrm{FEV}_{1}$, Einsekundenkapazität), und die nach Inhalation des Bronchodilatators beobachtete Zunahme des $\mathrm{FEV}_{1}$ wird als absolute oder relative Änderung im Verhältnis zum Ausgangswert dargestellt [15]. Die relative Änderung hängt naturgemäß von der Höhe der Ausgangswerte ab. Aus diesem Grunde wird empfohlen, dass die Kriterien zur Definition einer positiven Antwort sowohl einen relativen (z.B. um $12 \%$ [14] oder 15\% [12]) als auch einen absoluten Anstieg (z. B. um $200 \mathrm{~mL}$ ) beinhalten. Die Änderung des $\mathrm{FEV}_{1}$ nach Inhalation eines Bronchodilatators ist bei Patienten mit COPD meist gering ausgeprägt $[16,17]$, so dass die Atemwegsobstruktion als „irreversibel“ bezeichnet wird.

Ungeachtet dieses Zusammenhanges stellt sich die Frage, ob das geringe Ansprechen nicht teilweise durch die Wahl inadäquater Messparameter bedingt ist, zumal trotz der geringen Änderungen des $\mathrm{FEV}_{1}$ Bronchodilatatoren bei Patienten mit COPD regelhaft eingesetzt werden und in der Mehrzahl der Fälle zu einer merklichen symptomatischen Besserung führen $[18,19]$. Die positiven Wirkungen zeigen sich auch darin, dass nach Inhalation eines Bronchodilatators bei Patienten mit COPD die 6-Minuten-Gehstrecke zunimmt und die Dyspnoe abnimmt, unabhängig von der Änderung des $\mathrm{FEV}_{1}[20]$. Daher werden die klinischen Wirkungen durch die forcierte Exspiration nur ungenügend erfasst. Dies bestätigt auch der Befund, dass die 6-Minuten-Gehstrecke und die Dyspnoe vom $\mathrm{FEV}_{1}$ statistisch unabhängige Variable sind [21]. Aus den genannten Gründen sind Lungenfunktionsparameter vorzuziehen, welche mit den symptomatischen Änderungen korrelieren, um den individuellen Nutzen der Therapie zu objektivieren.

Bei der Suche nach validen Parametern zur Erfassung der Wirkung von Bronchodilatatoren bei Patienten mit COPD wandte sich die Aufmerksamkeit in der letzten Zeit den inspiratorischen Parametern zu. Bei Patienten mit COPD nimmt unter Belastung bekanntlich die Überblähung zu. Da sich die totale Lungenkapazität nicht ändert, ist die Zunahme 

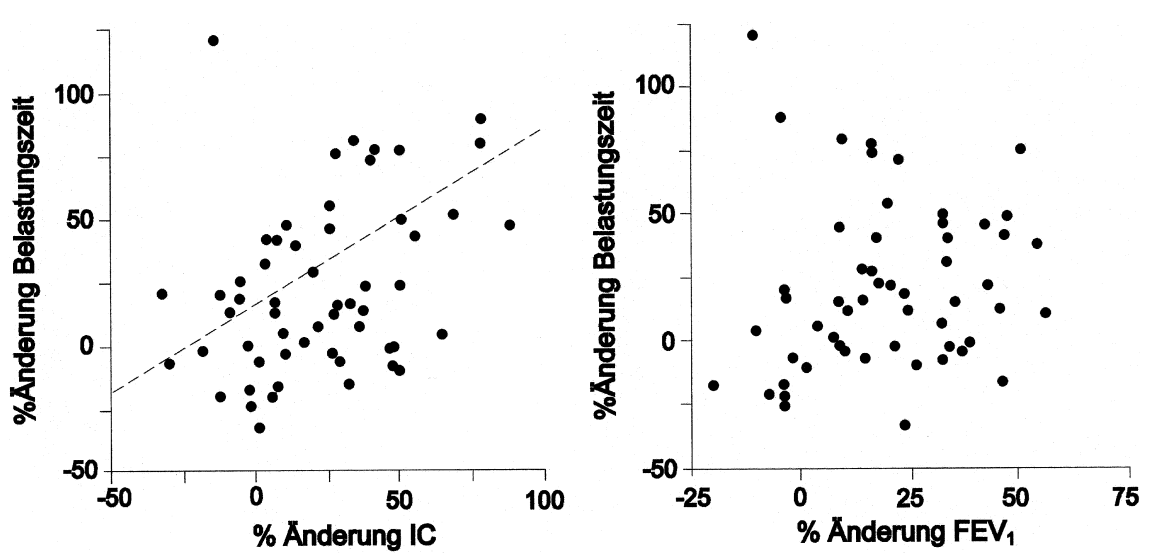

Abb. 1 Prozentuale Änderungen von inspiratorischer Kapazität (IC) und $\mathrm{FEV}_{1}$ relativ zum Ausgangswert im Vergleich zur Änderung der Belastungszeit in einem Ausdauertest nach Inhalation eines Bronchodilatators bei Patienten mit COPD. Während die Zunahme der IC mit dem Anstieg der Belastungszeit in Verbindung steht $(r=0,276$, $\mathrm{p}<0,05)$, besteht kein Zusammenhang zwischen der Änderung des $\mathrm{FEV}_{1}$ und der Änderung der Belastbarkeit $(r=0,215$, $\mathrm{p}=0,11)$. (Aus Referenz 26, mit Erlaubnis.) der Lungenüberblähung mit einer Abnahme der inspiratorischen Kapazität (IC) verbunden. Diese Abnahme, als Ausdruck der dynamischen Überblähung, korreliert mit dem Ausmaß der Belastungsdyspnoe [22,23]. Bronchodilatatoren verringern bei Patienten mit COPD die dynamische Überblähung unter Belastung signifikant [24-26]. Gleichzeitig wird die Belastungsdyspnoe reduziert und die körperliche Leistungsfähigkeit gesteigert. Die Zunahme der Leistungsfähigkeit ist dabei unabhängig von den Änderungen des FEV , während sie mit der Zunahme der IC sowohl in Ruhe (Abb.1) als auch unter Belastung korreliert [26]. Allerdings ist die Messung der dynamischen Überblähung nur während einer Spiroergometrie möglich und, verglichen mit einer konventionellen Broncholyse, relativ aufwendig. Sie wird daher in der Praxis nicht regelhaft angewandt.

Bei Patienten mit schwergradiger COPD ist meist eine exspiratorische Flusslimitierung (EFL) vorhanden, d.h. der exspiratorische Fluss ist bereits in Ruheatmung maximal [27]. Besteht eine EFL in Ruhe, kann der exspiratorische Fluss nur dann steigen, wenn man bei höheren Lungenvolumina atmet, so dass eine dynamische Überblähung entsteht. Die EFL kann mittels Anwendung eines Unterdruckes am Mund objektiviert werden [28]. In einer Untersuchung von Tantucci u. Mitarb. [29] führte die Inhalation eines Bronchodilatators bei Patienten mit EFL zu einer Abnahme der Überblähung, ausgedrückt in einer signifikanten Zunahme der IC. Bei den untersuchten Patienten ohne EFL war keine wesentliche Änderung der IC nachzuweisen. Jedoch fand sich keine Beziehung zwischen den Änderungen der IC in Ruhe und dem Dyspnoeempfinden.

Auch bezüglich anderer Lungenfunktionsparameter konnte bei Patienten mit COPD bislang kein Zusammenhang zwischen Dyspnoeempfinden und lungenfunktionellen Veränderungen nachgewiesen werden $[21,30,31]$, im Gegensatz zu Patienten mit Asthma bronchiale, bei denen die Änderung der Dyspnoe eng mit den Änderungen des $\mathrm{FEV}_{1}$ korreliert [18]. Einigen Untersuchern fiel auf, dass bei Patienten mit COPD die symptomatischen Änderungen nach Inhalation eines Bronchodilatators heterogen sind. Noseda u. Mitarb. differenzierten zwei Gruppen $[18,32]$ : die eine zeigte nach Inhalation des Bronchodilatators eine deutliche Abnahme im Dyspnoeempfinden („high perceivers“), während die andere keine wesentliche Änderung der Beschwerdesymptomatik angab („low perceivers“). Da sich in beiden Gruppen die exspiratorischen Lungenfunktionsparameter $\left(\mathrm{FEV}_{1}\right.$, mittlere exspiratori- sche Flussrate bei 50\% der Vitalkapazität) nur unwesentlich änderten und kein Bezug zur Änderung der Dyspnoe bestand (Abb. 2), wurden die Unterschiede auf solche der Perzeption zurückgeführt. In beiden Gruppen zeigten jedoch die Änderungen der Parameter der Inspiration (inspiratorische Vitalkapazität, inspiratorischer Widerstand, mittlere inspiratorische Flussrate bei 50\% der Vitalkapazität) einen signifikanten Unterschied.

Die exspiratorische Flussbegrenzung bei Patienten mit COPD wird im Wesentlichen auf strukturelle Änderungen der Atemwege zurückgeführt [33], die den Kollaps der Atemwege begünstigen [9]. Dieser Kollaps tritt auch dann auf, wenn der Tonus der Atemwege durch Relaxation der glatten Atemwegsmuskulatur reduziert wird. In-vitro-Untersuchungen an isolierten Atemwegen von Rauchern lassen vermuten, dass die Kollapsibilität der Atemwege nach Relaxation sogar zunimmt [34]. Diese Befunde legen die Hypothese nahe, dass bei Patienten mit COPD eine Verringerung des Tonus der Atemwegsmuskulatur während forcierter exspiratorischer Manöver nicht zutagetreten kann, da die Flussbegrenzung dominiert und die Relaxation maskiert. Im Gegensatz dazu verhindert während einer forcierten Inspiration der negative intrapleurale Druck den Kollaps der Atemwege, so dass die Bronchodilatation zutage tritt. Diese Überlegung legt nahe, dass bei der COPD die Effekte inhalierter Bronchodilatatoren während forcierter inspiratorischer Manöver ausgeprägter sein sollten als während forcierter exspiratorischer Manöver. Die Hypothese impliziert auch, dass die Abnahme der Dyspnoe auf die Erleichterung der Inspiration zurückzuführen sein könnte. Eine derartige Erklärung würde die angeführte Diskrepanz zwischen „negativem Broncholysetest“ und positiven klinischen Wirkungen auflösen.

Ausgehend von diesen Überlegungen untersuchten wir die Änderungen inspiratorischer und exspiratorischer Lungenfunktionsparameter sowie den Zusammenhang zwischen den Änderungen dieser Parameter und den Änderungen des Dyspnoeempfindens bei insgesamt 61 Patienten mit COPD verschiedenen Schweregrades [35]. Zur Beschreibung der Daten wurde der statistische Ansatz der Faktorenanalyse gewählt, der sich in vorangegangenen Untersuchungen bereits bewährt hatte $[21,30,31]$. Nach Inhalation eines $\beta_{2}$-Sympathomimetikums stiegen im Mittel die Werte sowohl der forcierten exspiratorischen $\left(\mathrm{FEV}_{1}\right)$ als auch der forcierten inspiratorischen $\left(\mathrm{FIV}_{1}\right)$ Parameter an. Der Anstieg war in den inspirato- 

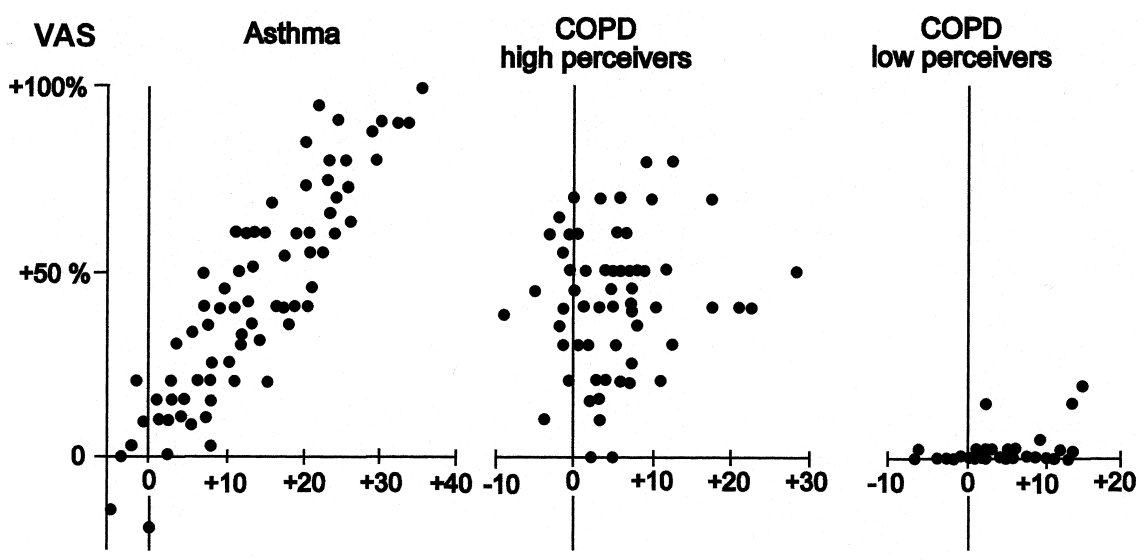

Abb. 2 Zusammenhang zwischen der prozentualen Änderung des FEV 1 und der Änderung der Dyspnoe erfasst durch eine visuelle Analogskala (VAS) nach Applikation eines Bronchodilatators. Bei Patienten mit Asthma bronchiale besteht ein klarer Zusammenhang zwischen der Änderung der Dyspnoe und des FEV 1 . Dahingehend ist sowohl bei Patienten mit COPD, die eine starke Änderung des Dyspnoeempfindens nach Applikation eines Bronchodilatators aufweisen („high perceiver“), als auch solchen, die keine wesentliche Änderung des Dyspnoeempfindens zeigen („low perceiver"), kein Zusammenhang mit den Änderungen des $\mathrm{FEV}_{1}$ zu finden. (Aus Referenz 18 , mit Erlaubnis.)

$\mathrm{FEV}_{1} \%$ Änderung vom Ausgangswert

rischen Parametern ausgeprägter als in den exspiratorischen Parametern, insbesondere bezüglich der absoluten Werte (Abb.3). Auch nahmen das intrathorakale Gasvolumen und das Residualvolumen nach Inhalation des Bronchodilatators ab. Ungeachtet der gleichgerichteten Änderungen der Mittelwerte dieser Parameter zeigte allein das forcierte inspiratorische Volumen in einer Sekunde $\left(\right.$ FIV $\left._{1}\right)$ eine enge Beziehung zur Dyspnoe, während die Korrelation mit IC, FEV , PEF, ITGV und RV wesentlich geringer war. Die Faktorenanalyse bestätigte, dass die Reduktion der Dyspnoe nach Broncholyse überwiegend mit der Verbesserung des FIV $_{1}$ und in geringerem Maße mit derjenigen der inspiratorischen Vitalkapazität und des PIF zusammenhing. Diese Ergebnisse zeigen, dass bei Patienten mit COPD die forcierte Inspiration, insbesondere die Messung des FIV $_{1}$, diejenigen objektiven Messgrößen liefert, welche die Änderungen des subjektiven Dyspnoeempfindens

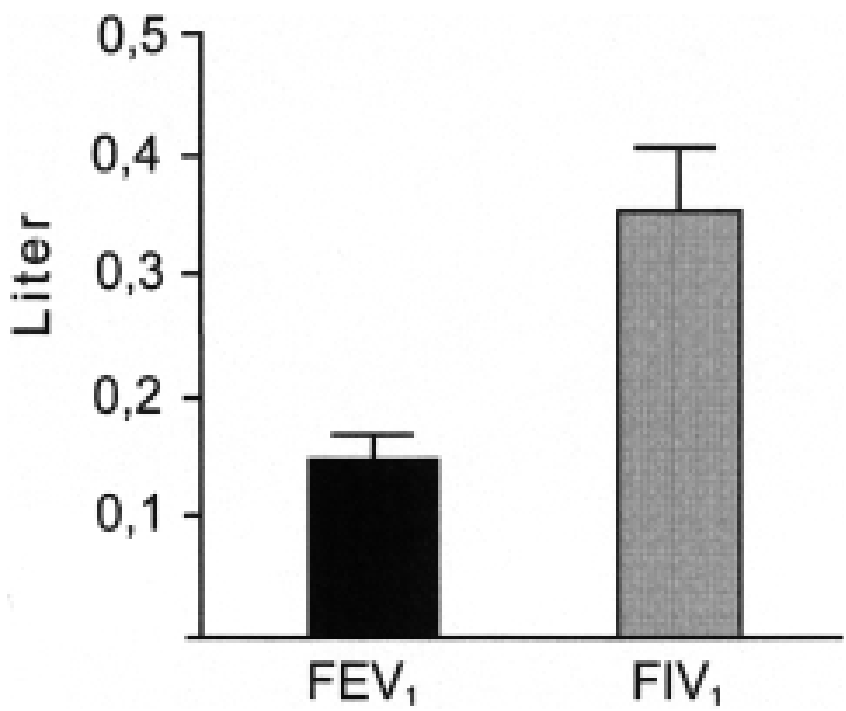

Abb. 3 Änderung der Lungenfunktion (Mittelwert und Standardfehler) nach Applikation eines Bronchodilatators bei Patienten mit mittel- bis schwergradiger COPD. Nach Inhalation des $\beta_{2}$-Sympathomimetikums stiegen im Mittel die Werte sowohl der forcierten exspiratorischen $\left(\mathrm{FEV}_{1}\right)$ als auch der forcierten inspiratorischen $\left(\mathrm{FIV}_{1}\right)$ Parameter an. Der Anstieg war in den inspiratorischen Parametern ausgeprägter als in den exspiratorischen Parametern. nach Inhalation eines Bronchodilatators erklären können. Dies impliziert, dass die in der Literatur getroffene Unterscheidung zwischen "high" und „low perceivers“, die sich vornehmlich auf die mangelhafte Korrelation zwischen Dyspnoe und $\mathrm{FEV}_{1}$ stützt, zu überdenken ist. Der Atemstoß $\mathrm{FEV}_{1}$ ist offenbar bei Patienten mit COPD zur Erfassung des Dyspnoeempfindens nicht geeignet. Die Plausibilität dieser Überlegungen und Daten wird durch die Tatsache unterstützt, dass viele Patienten angeben, durch die Bronchodilatatoren „leichter Luft zu bekommen“.

Die Analyse der Literatur und der neuen Erkenntnisse legen nahe, dass die nach wie vor zur Quantifikation des Broncholysetestes verwandte forcierte Exspiration für die Beurteilung der Wirkung von Bronchodilatatoren bei Patienten mit COPD nur bedingt geeignet ist. Dies ist vermutlich auf den für dieses Krankheitsbild kennzeichnenden dynamischen Kollaps der Atemwege während Exspiration zurückzuführen. Daher erscheinen solche Methoden der Lungenfunktionsmessung von primärem Interesse, die entweder das Ausmaß des Kollapses durch Anwendung eines negativen exspiratorischen Druckes erfassen oder aber den Kollaps durch forcierte inspiratorische Manöver umgehen. Diese Verfahren zeigen im allgemeinen größere Effekte inhalierter Bronchodilatatoren als die forcierte Exspiration und lassen verstehen, warum viele Patienten mit COPD ungeachtet der Irreversibilität ihrer Atemwegsobstruktion, gemessen am $\mathrm{FEV}_{1}$, von einer solchen Behandlung profitieren. Weitere Untersuchungen werden zeigen, inwieweit die akut gemessenen funktionellen Verbesserungen und die damit verbundene Reduktion der Dyspnoe auch über den langfristigen Nutzen eines Bronchodilatators bei einem individuellen Patienten zu entscheiden erlauben.

\section{Literatur}

${ }^{1}$ Wiesner B. Epidemiologie chronisch-obstruktiver Atemwegserkrankungen. Atemw.-Lungenkrankheiten 1992; 18: 75 - 78

${ }^{2}$ ERS Consensus Statement. Optimal assessment and management of chronic obstructive pulmonary disease (COPD). Eur Respir J 1995; 8: $1398-1420$

${ }^{3}$ American Thoracic Society. Standards for the diagnosis and care of patients with chronic obstructive disease (COPD) and asthma. Am J Respir Crit Care Med 1995; 152: 77-120 
${ }^{4}$ Lange P, Parner J, Vestbo P, Schnohr P, Jensen G. A 15-year follow-up study of ventilatory function in adults with asthma. N Eng J Med 1998; 339: 1194-1200

${ }^{5}$ Muller NL, Stapels CA, Miller RR, Abboud RT. „Density mask“. An objective method to quantitate emphysema using computed tomography. Chest 1988; 94: $782-787$

${ }^{6}$ Kohlhäufel M, Brand P, Selzer T, Scheuch G, Meyer T, Weber N, Schulz K, Häussinger K, Heyder J. Diagnosis of emphysema in patients with chronic bronchitis: a new approach. Eur Respir J 1998; $12: 793-798$

${ }^{7}$ Magnussen $\mathrm{H}$, Richter K, Taube C. Are chronic obstructive pulmonary disease (COPD) and asthma different diseases? Clin Exper Allergy 1998; 28, Suppl. 5: 187-194

${ }^{8}$ Thurlbeck WM. Pathology of chronic airflow obstruction. Chest 1990; 97: S6-S10

${ }^{9}$ Leaver DG, Tattersfield AE, Pride NB. Contributions of loss of elastic recoil and of enhanced airways collapsibility to the airflow obstruction of chronic bronchitis and emphysema. J Clin Invest 1973; 52: $2117-2128$

10 Saetta M, Kim WD, Izquierdo JL, Ghezzo H, Cosio MG. Extent of centrilobular and panacinar emphysema in smokers' lung: pathological and mechanical implications. Eur Respir J 1994; 7: 664-671

11 Brand PLP, Postma DS, Kerstjens HAM, Koeter GH and the Dutch CNSLD Study Group. Relationship of airway hyperresponsiveness to respiratory symptoms and dinural peak flow variation in patients with obstructive lung disease. Am Rev Respir Dis 1991; 143: $916-921$

12 British Thoracic Society. Guidelines for the management of COPD. Thorax 1997; 52: S1 - S28

${ }^{13}$ Wettengel R, Böhning W, Cegla U, Criée C, Fichter J, Geisler L, Fabel H, Köhler D, Konietzko N, Lindemann H, Magnussen H, Matthys $H$, Meister R, Morr $H$, Nolte D, Petro W, SchulzeWerninghaus G, Sill V, Sybrecht G, Wiesner B, Worth H. Empfehlungen der Deutschen Atemwegsliga zur Behandlung von Patienten mit chronisch obstruktiver Bronchitis und Lungenemphysem. Med Klin 1995; 90: $3-7$

14 American Thoracic Society. Lung function testing: Selection of reference values and interpretative strategies. Am Rev Respir Dis 1991; 144: $1202-1228$

15 Brand PLP, Quanjer PH, Postma DS, Kerstjens HAM, Koëter GH, Dekhuijzen PNR, Sluiter HJ and the Dutch chronic non-specific lung disease (CNSLD) study group. Interpretation of bronchodilator response in patients with obstructive airway disease. Thorax 1992; 47: 429-430

${ }^{16}$ Anthonisen NR, Wright EC and the IPPB Trial Group. Bronchodilator response in chronic obstructive pulmonary disease. Am Rev Respir Dis 1986; 133: 814-819

${ }^{17}$ Nisar M, Harris JE, Pearson MG, Calverley PMA. Acute bronchodilator trials in chronic obstructive pulmonary disease. Am Rev Respir Dis 1992; 146: 555 - 559

${ }^{18}$ Noseda A, Schmerber J, Prigogine T, Yernault JC. How do patients with either asthma or COPD perceive acute bronchodilatation? Eur Respir J 1993; 6: 636-644

${ }^{19}$ Gibson GJ, MacNee W. Chronic obstructive pulmonary disease: investigation and assessment of severity. Eur Respir Mon 1998; 7: $25-40$

${ }^{20}$ Hay JG, Stone P, Carter J, Church S, Eyre Brook A, Pearson MG, Woodcock AA, Calvereley PMA. Bronchodilator reversibility, exercise performance and breathlessness in stable chronic obstructive pulmonary disease. Eur Respir J 1992; 5: 659-664

${ }^{21}$ Wegner RE, Jörres RA, Kirsten DK, Magnussen H. Factor analysis of exercise capacity, dyspnoea ratings and lung function in patients with severe COPD. Eur Respir J 1994; 7: 725 - 729
22 O'Donnell DE, Webb KA. Exertional breathlessness in patients with chronic airflow limitation: the role of hyperinflation. Am Rev Respir Dis 1993; 148: 1351 - 1357

23 O’Donnell DE, Bertley JC, Chau LK, Webb KA. Qualitative aspects of exertional breathlessness in chronic airflow limitation: pathophysiologic mechanisms. Am J Respir Crit Care Med 1997; 155: $109-115$

24 O’Donnell DE, Lam M, Webb KA. Measurement of symptoms, lung hyperinflation, and endurance during exercise in chronic obstructive pulmonary disease. Am J Respir Crit Care Med 1998; 158: $1557-1565$

${ }^{25}$ Belman MJ, Botnick WC, Shin JW. Inhaled bronchodilators reduce dynamic hyperinflation during exercise in patients with chronic obstructive pulmonary disease. Am J Respir Crit Care Med 1996; 153: 967-975

${ }^{26}$ O’Donnell DE, Lam M, Webb KA. Spirometric correlates of improvement in exercise performance after anticholinergic therapy in chronic obstructive pulmonary disease. Am J Respir Crit Care Med 1999; 160: 542-549

${ }^{27}$ Koulouris NG, Valta P, Lavoie A, Corbeil C, Chasse M, Braidy M, Milic-Emili J. A simple method to detect exspiratory flow limitation during spontaneous breathing. Eur Respir J 1995; 8: $306-$ 313

${ }^{28}$ Valta P, Corbeil C, Lavoie A, Cammpodonico R, Koulouris N, Chasse M, Braidy J, Milic-Emili J. Detection of expiratory flow limitation during mechanical ventilation. Am J Respir Crit Care Med 1994; 150: 1311 - 1317

${ }^{29}$ Tantucci C, Duguet A, Similowski T, Zelter M, Derenne JP, MilicEmili J. Effect of salbutamol on dynamic hyperinflation in chronic obstructive pulmonary disease patients. Eur Respir J 1998; 12: 799-804

${ }^{30}$ Ries AL, Kaplan RM, Blumberg E. Use of factor analysis to consolidate multiple outcome measures in chronic obstructive pulmonary disease. J Clin Epidemiol 1991; 44: 497-503

${ }^{31}$ Mahler DA, Harver A. A factor analysis of dyspnea ratings, respiratory muscle strength and lung function in patients with chronic obstructive pulmonary disease. Am Rev Respir Dis 1992; 145: $467-470$

${ }^{32}$ Noseda A, Schmerber J, Prigogine T, de Maertelaer V, Yernault JC. Perception of dyspnoea during acute changes in lung function in patients with either asthma or COPD. Respir Med 1995; 89: 477-485

${ }^{33}$ Paré PD, Armour C, Taylor S, Mullen B, Moreno R, Hogg JC, Schellenberg R. Airway hyperreactivity in COPD cause or effect, an in vivo in vitro comparison. Chest 1987; 91: S40-S44

${ }^{34}$ Tiddens HAWM, Hofhuis W, Bogaard JM, Hop WCJ, de Bruin $\mathrm{H}$, Willems LNA, de Jongste JC. Compliance, hysteresis, and collapsibility of human small airways. Am J Respir Crit Care Med 1999; 160: $1110-1118$

${ }^{35}$ Taube C, Lehnigk B, Paasch K, Kirsten DK, Jörres RA, Magnussen $\mathrm{H}$. Factor analysis of changes in dyspnea and lung function parameters after bronchodilation in COPD. Am J Respir Crit Care Med im Druck

\section{Dr. med. C. Taube}

Pneumologisches Forschungsinstitut am Krankenhaus Großhansdorf

Zentrum für Pneumologie und Thoraxchirurgie

Wöhrendamm 80

22927 Großhansdorf

E-mail: c.taube@pulmoresearch.de 